\title{
Erratum: identifying the neutrino mass ordering with INO and NOvA
}

\section{Mattias Blennow and Thomas Schwetz}

Max-Planck-Institut für Kernphysik,

Saupfercheckweg 1, 69117 Heidelberg, Germany

E-mail: blennow@mpi-hd.mpg.de, schwetz@mpi-hd.mpg.de

ERratum TO: JHEP08(2012)058

There is a typo in Equation (2.2) of our paper [1]. The correct form of the equation is

$$
\Delta m_{\text {eff }}^{2}=\Delta m_{31}^{2}-\left(\cos ^{2} \theta_{12}-\cos \delta \sin \theta_{13} \sin 2 \theta_{12} \tan \theta_{23}\right) \Delta m_{21}^{2},
$$

as originally presented in [2] [equation (17)]. Our numerical simulations and results remain unchanged as they were implemented using the correct form of the equation.

\section{References}

[1] M. Blennow and T. Schwetz, Identifying the neutrino mass ordering with INO and NOvA, JHEP 08 (2012) 058 [arXiv:1203.3388] [INSPIRE].

[2] H. Nunokawa, S.J. Parke and R. Zukanovich Funchal, Another possible way to determine the neutrino mass hierarchy, Phys. Rev. D 72 (2005) 013009 [hep-ph/0503283] [INSPIRE]. 